\title{
Por que a Letalidade Hospitalar do Infarto Agudo do Miocárdio é Maior nas Mulheres?
}

\author{
Luiz Carlos Santana Passos, Antonio Alberto Lopes, A mine A morim Barbosa, Rogério Santos Jesus
}

São Paulo, SP

Objetivo - Avaliar a influência da idade, da gravidade da doença e das intervenções terapêuticas na maior letalidade hospitalar do infarto agudo do miocárdio (IAM) em mulheres.

Métodos - Estudo de coorte retrospectivo, envolvendo 388 pacientes com IAM (50 óbitos). Foram avaliadas, como possíveis explicações para a associação entre sexo e letalidade hospitalar do IAM, as variáveis: idade ( $<60 \mathrm{vs}$ $\geq 60$ anos), duração de sintomas, classe de Killip, tipo de IAM (com ou sem ondas $Q$ ), comorbidades, história de acidente vascular cerebral e intervenções terapêuticas para o IAM (ácido acetil-salicílico, betabloqueadores e agentes trombolíticos). Modelos de regressão logística foram usados para avaliar a influência de potenciais variáveis confundidoras na associação entre sexo e letalidade hospitalar do IAM.

Resultados - A letalidade hospitalar do IAM foi mais alta em mulheres $(19,5 \%$ vs 9,4\%) do que em homens (odds ratio $(O R)=2,34$; IC 95\% = 1,12-4,47). Embora as mulheres fossem significantemente ( $p<0,01)$ mais idosas, a associação entre sexo e morte reduziu-se em apenas $15 \%$ após ajuste para idade $(O R=1,99 ;$ IC $95 \%=1,07$ 3,67). Esta associação tornou-se mais fraca ao se considerar a gravidade da doença na admissão $(O R=1,84 ;$ IC $95 \%=0,90-3,74)$ e intervenções terapêuticas para $o$ IAM $(O R=1,50 ; I C=0,67-3,38)$.

Conclusão - Diferenças de idade não podem explicar completamente a maior letalidade do IAM em mulheres. A gravidade da doença na admissão e diferenças de abordagem terapêutica devem desempenhar importante papel na maior letalidade hospitalar do IAM em mulheres.

Palavras-chave infarto agudo do miocárdio, letalidade hospitalar, mulheres

\section{Why is the In-Hospital Case-Fatality Rate of Acute Myocardial Infarction Higher in Women?}

Purpose - To assess the influence of age, disease severity at admission and therapeutic interventions on the higher in-hospital case-fatality rate of acute myocardial infarction (MI) in women.

Methods - A retrospective cohort study involving a total of 388 acute MI patients (50 deaths). The following variables were treated as possible explanatory factors for the association between gender and case-fatality rate of acute MI: age ( $<60 v s \geq 60$ years), duration of symptoms, Killip class, type of infarction ( $Q$ wave vs non- $Q$ wave), comorbidities, previous history of stroke and specific therapeutic interventions for acute MI (aspirin, betablocking drugs, and thrombolytic agents). Logistic regression models were used to assess the influence of potential confounders on the association between gender and in-hospital death.

Results - The in-hospital case-fatality rate of acute MI was higher in women (19.5\% vs 9.4\%) than in men (odds ratio $(O R)=2.34 ; 95 \% C I=1.12-4.47)$. Although women were significantly older than men $(p<0.01)$, the association between gender and death was reduced only by 15 percent after adjusting for age $(O R=1.99 ; 95 \% C I=$ 1.07-3.67). This association became weaker after taking into account disease severity at admission $(O R=1.84$; 95\% CI $=0.90-3.74)$ and therapeutic interventions for acute $M I(O R=1.50 ; 95 \% C I=0.67-3.38)$.

Conclusion - Differences in age cannot fully explain the higher case-fatality rate in women than in men with acute MI. Severity of disease at admission and differences in therapeutic interventions might play an important role in the higher case-fatality rate of acute MI among women.

Key-words: acute myocardial infarction, in-hospital casefatality rate, women

Arq Bras Cardiol, volume 70 (n 5), 327-330, 1998

Faculdade de Medicina da Universidade Federal da Bahia - Salvador Correspondência: Luiz Carlos Santana Passos - Rua Amir Macedo, 119/1003 40285-460 - Salvador, BA

Recebido para publicação em 25/9/97

Aceito em 4/3/98
Em contraste com a incidência do infarto agudo do miocárdio (IAM), maior entre homens, a letalidade hospitalar do IAM é maior entre as mulheres ${ }^{1-5}$. Em Salvador, o número de mortes por IAM vem crescendo desde o final dos 
anos 70, particularmente, entre as mulheres. No período de 1981-1994, o número de mortes por IAM entre os homens aumentou em $26,2 \%$, enquanto que para as mulheres o aumento foi de $46,4 \%{ }^{6}$.

Embora diversos estudos mostrem, de forma consistente, maior mortalidade em mulheres do que em homens com IAM, as razões para esse fenômeno não estão devidamente esclarecidas ${ }^{1-10}$. Estudos conduzidos fora do Brasil, no entanto, sugerem que a idade mais avançada, a maior gravidade da insuficiência ventricular esquerda(IVE) e fatores relacionados com à terapêutica, podem, em conjunto, contribuir para a maior letalidade do IAM entre as mulheres.

Foi citado anteriormente que, em Salvador, entre 19931994, a letalidade hospitalar do IAM foi aproximadamente duas vezes maior nas mulheres em relação aos homens ${ }^{11}$, concordando, portanto, com o que tem sido observado em diversos outros lugares. As possíveis razões para a maior mortalidade em mulheres, no entanto, não foram exploradas.

Esse trabalho avalia possíveis efeitos, independentes ou associados, da idade, da gravidade da IVE e de intervenções terapêuticas na maior letalidade hospitalar, observada em mulheres, no curso do IAM.

\section{Métodos}

Estudo de coorte retrospectivo baseado em prontuários médicos de seis dos principais hospitais públicos e privados da cidade de Salvador. De 1/1/93 e 31/12/94, foram revistos 688 prontuários de pacientes com suspeita de IAM, no momento da admissão, sendo que o diagnóstico foi confirmado em $451(65,6 \%)$ casos, através de critérios clínicos (dor torácica >30min), eletrocardiográficos (supradesnível ou infradesnível do segmento ST) e enzimáticos (elevação de pelo menos duas vezes o valor normal da isoenzima cardíaca creatininofosfoquinase). A análise restringiu-se aos $388(86,0 \%)$ pacientes que, além do diagnóstico confirmado de IAM, apresentaram tempo de sintomas <24h. Os hospitais públicos participaram com $163(42 \%)$ pacientes, os filantrópicos com 88 (22,7\%) e os privados com 137 (34,3\%). A coleta de dados foi realizada através de questionário-padrão. As variáveis preditoras incluíram: 1) características demográficas (sexo, idade); 2) características clinicas presumivelmente relacionadas com a gravidade do IAM (tempo de sintomas, classe de Killip, tipo do infarto (com e sem onda Q) e comorbidades) e 3) intervenções terapêuticas para IAM (ácido acetilsalicílico, betabloqueadores, e agentes trombolíticos). Outros detalhes sobre a coleta de dados, operacionalização das variáveis e características da amostra foram apresentados em estudo anterior ${ }^{11}$.

Os testes do qui quadrado (ou o teste exato de Fisher) e t de Student (ou o teste de Mann Whitney) para amostras independentes foram utilizados para determinar a significância estatística de diferenças entre proporções e médias, respectivamente, após verificar se os dados eram consistentes com as suas premissas. A odds ratio (OR) foi usada como medida de associação por ser essa medida e respectivos intervalos de confiança (IC) facilmente determina- dos, através dos antilogaritmos dos coeficientes da regressão logística múltipla ${ }^{12}$.

A necessidade de modelos logísticos estratificados para diferentes níveis de variáveis independentes foi avaliada através da significância estatística dos coeficientes de interação entre a variável sexo e cada uma das outras covariáveis. Como nenhuma das interações foi estatisticamente significante, as variáveis preditoras puderam ser incluídas conjuntamente nos modelos. Os procedimentos frequencies, crosstabs, t-test e logistic regression do Statistical Package for Social Science, SPSS, foram usados nas análises estatísticas ${ }^{13,14}$. Para avaliar a significância estatística das associações, a hipótese nula foi de $\mathrm{OR}=1$. Todos os testes foram bicaudais e o nível de significância estatística foi pré-estabelecido em $5 \%$.

\section{Resultados}

Em relação às características clínicas obtidas na admissão, as mulheres eram 6,3 anos mais idosas do que os homens, apresentavam mais freqüentemente classe de Killip $>1$ e mais infartos subendocárdicos (tab. I). As mulheres também chegaram mais tardiamente às unidades hospitalares, apresentaram mais comorbidades e maior freqüência de história prévia de acidente vascular cerebral (AVC), embora essas diferenças não alcançassem significância estatística.

O emprego de agentes trombolíticos e betabloqueadores, intervenções farmacológicas consideradas eficazes no IAM, foi maior entre os homens do que entre as mulheres. O uso do ácido acetilsalicílico no entanto, foi similar entre os sexos (tab. I).

A letalidade hospitalar do IAM foi de 19,6\% (26/133) em mulheres e 9,4\% (25/255) em homens ( $\mathrm{p}<0,05)$, correspondendo a uma OR de 2,34; IC 95\%=1,12-4,47 (fig. 1). Quan-

\begin{tabular}{|lccc|}
\hline \multicolumn{4}{|c|}{ Tabela I - Características clínicas de 388 pacientes com IAM de acordo } \\
com sexo em Salvador (1993/1994)
\end{tabular}




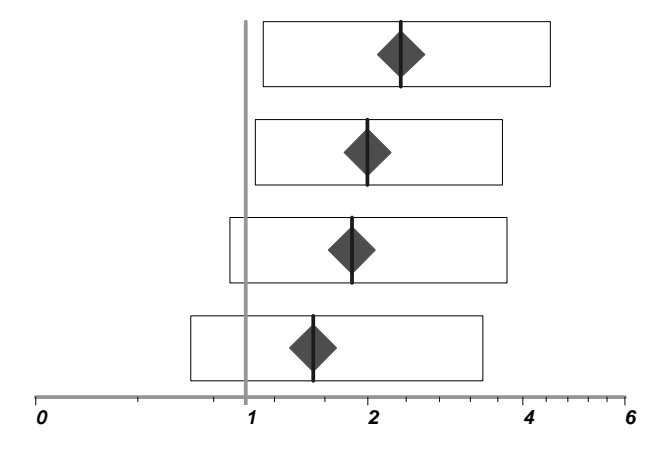

\author{
$\mathrm{OR}^{*}$ não ajustada $=2,34$ \\ (IC95\%=1,12-4,47) \\ OR ajustada para idade $=1,99$ \\ IC95\%=1,07-3,67) \\ OR ajustada para variáveis de gravidade $=1,84$ \\ (IC95\% $=0,90-3,74)$ \\ OR após inclusão de variáveis de tratamento=1,50 \\ $($ IC95\% $=0,67-3,38)$
}

Fig. 1 - Associação entre sexo (mulheres vs homens) e letalidade hospitalar do IAM. * Odds ratio da associação entre sexo e mortalidade

do se realizou ajuste para a variável idade (modelo logístico incluindo apenas as variáveis sexo e idade como preditoras), a OR reduziu-se em $15 \%$ (OR $=1,98$; IC $95 \%=1,07$ $3,66)$ e permaneceu estatisticamente significante $(\mathrm{p}<0,05)$. A associação entre sexo e letalidade sofreu uma redução adicional de $7 \%(\mathrm{OR}=1,84 ;$ IC $95 \%=0,90-3,74)$ ao se considerar a gravidade do IAM. No modelo completo (i.e., incluindo sexo, idade, variáveis de gravidade e intervenções terapêuticas) a associação entre sexo e letalidade ( $\mathrm{OR}=1,50$; IC $95 \%=0,67-3,38$ ) tornou-se ainda menos intensa (fig. 1 ).

\section{Discussão}

No presente estudo foi observado que o maior risco de morte durante a fase hospitalar do IAM entre as mulheres pode ser explicado, pelo menos parcialmente, por diferenças na apresentação clínica e na abordagem terapêutica. Os dados apóiam o papel da idade como preditor independente de morte no IAM, o que está de acordo com os resultados de estudos anteriores ${ }^{3,7-9}$. A idade mais avançada das mulheres, no entanto, explica apenas uma pequena parte das diferenças entre os sexos na letalidade do IAM.

Ao se acrescentar a classe de Killip e outras variáveis indicadoras de gravidade, ao modelo de regressão logística múltipla, detectou-se uma redução mais acentuada nas diferenças de mortalidade, entre os sexos, quando comparado ao modelo que incluiu apenas a idade. Isto apóia a idéia de que a maior gravidade do evento coronário entre as mulheres, não pode ser simplesmente explicado pelo fato de que elas são, no momento do IAM, mais idosas do que os homens.

As razões para a maior freqüência de IVE (i.e, classe de Killip>I) na fase aguda do IAM, entre as mulheres, não são claras ${ }^{2,3,15,16}$. Existem evidências, no entanto, de que o padrão hemodinâmico da IVEé diferente entre os sexos. Por exemplo, as mulheres têm mais disfunção diastólica do VE do que os homens no curso do IAM ${ }^{17}$, o que pode contribuir para a maior mortalidade das mulheres na fase aguda do evento, sem comprometer a sobrevida a longo prazo, a qual está mais relacionada com a disfunção ventricular esquerda do tipo sistólica ${ }^{18}$.

Um achado intrigante foi observado em uma análise post hoc do presente estudo. As mulheres com infarto subendocárdico, infartos geralmente de menor extensão, apresentaram letalidade $(14,4 \%)$ maior do que homens com infarto transmural $(10,6 \%)$. Esses achados favorecem a hipótese de que as diferenças entre os sexos são, pelo menos em parte, decorrentes de mecanismos biológicos. Uma análise detalhada do grau e padrão da IVE, bem como da intensidade das lesões obstrutivas das coronárias, através da ecocardiografia e cineangiocoronariografia na fase aguda do IAM, irá ajudar a estabelecer o papel de fatores estruturais e funcionais na maior letalidade hospitalar do IAM em mulheres.

A maior frequiência de AVC prévio em pessoas do sexo feminino, observada neste e em outros estudos ${ }^{19}$, poderia sugerir um maior grau de ateroesclerose entre as mulheres. Pelo menos, no que diz respeito às artérias coronárias, as evidências apontam em sentido oposto. Estudos com cinecoronariografia na fase aguda do IAM mostram uma menor intensidade de aterosclerose coronária obstrutiva em mulheres, seja na presença de IAM ou de angina instável ${ }^{17,20}$. Esses achados reforçam a hipótese de que o desenvolvimento do IAM pode seguir caminhos patogenéticos diferentes entre homens e mulheres.

O menor uso de terapia trombolítica e de betabloqueadores em mulheres encontrado neste estudo é condizente com relatos prévios ${ }^{21-24}$. Os dados apóiam a hipótese de que as diferentes condutas terapêuticas podem ser, em parte, decorrentes das características clínicas das mulheres no momento da admissão. Não se pode afastar a possibilidade, no entanto, de que estes achados sejam o resultado de idéias preconcebidas dos médicos, em relação ao IAM em mulheres. A maior ocorrência de infartos subendocárdicos (em que a terapia trombolítica não está indicada), entre as mulheres, é um importante fator limitante para ampliação do uso de fibrinolíticos neste grupo. Além do mais, a idade mais avançada e a maior freqüência de comorbidades podem justificar a menor freqüência de trombólise entre as mulheres.

A maior frequiência de IVE, no momento da admissão, em mulheres, deve ter contribuído para limitar o uso mais amplo de betabloqueadores neste grupo. Considerando que estes agentes estão contra-indicados de forma absoluta, apenas para pacientes com disfunção sistólica e que o padrão hemodinâmico da IVE difere entre os sexos, torna-se 
necessário rever a adequação da restrição deste grupo de drogas em mulheres. Este ponto merece ser enfocado em estudos futuros visto que os betabloqueadores têm eficácia comprovada na redução da mortalidade do IAM. O uso mais freqüente de estudo ecocardiográfico no momento da admissão, ao contribuir para identificar o padrão de IVE, poderia ajudar a identificar a terapêutica mais adequada e, assim, reduzir as diferenças de letalidade do IAM entre os sexos.

O tipo de terapêutica escolhido para tratar pacientes com IAM tem sido apontado por diversos investigadores como um dos fatores que contribui para o pior prognóstico entre as mulheres ${ }^{15,19,24}$. Os resultados apresentados neste estudo, através da regressão logística, são de certa forma condizentes com esta possibilidade, visto que as diferenças de letalidade entre os sexos foram atenuadas ao se levar em consideração as variáveis de tratamento, mesmo com a manutenção das variáveis de gravidade no modelo.

Ao se procurar definir o papel da terapêutica nas diferenças de mortalidade entre os sexos, é importante observar que as mulheres têm sido subrepresentadas nos ensaios clínicos com terapia trombolítica. Ao lado disso, esses estudos experimentais têm incluído pacientes com risco de morte relativamente mais baixo do que aqueles selecionados para estudos epidemiológicos observacionais. É possível que novas intervenções e estratégias terapêuticas voltadas para pacientes de alto risco (freqüentemente inelegíveis para trombólise ou betabloqueadores) possam ter maior impacto na redução da diferença de letalidade do IAM entre os sexos. Dados preliminares sugerem que o uso preferencial da angioplastia primária acompanha-se de redução dessa diferença entre homens e mulheres no risco de morrer de IAM durante o período de hospitalização ${ }^{25}$.

Finalmente, é possível que outros fatores biológicos, psicossociais e comportamentais não avaliados neste estudo possam também contribuir para as diferenças de mortalidade entre os sexos. Entre essas variáveis devem ser incluídas: índice de massa corpórea, nível socioeconômico, freqüência de detecção e tratamento de doenças associadas, estresse psicológico e o comportamento do paciente na busca de cuidados médicos. O papel destes fatores, bem como a influência do tipo de intervenção terapêutica, nas diferenças de letalidade hospitalar do IAM entre homens e mulheres representam importantes questões a serem estudadas em investigações futuras.

\section{Agradecimentos}

Às equipes médicas e administrativas de todos os hospitais envolvidos neste estudo pela colaboração prestada.

\section{Referências}

1. Greenland P, Reicher-Reiss H, Goudbourt U, Behar S, Israeli SPRINT investigators - In-hospital and 1-year mortality in 1.524 women after myocardial infarction. Circulation 1991; 83: 484-91.

2. Fiebach NH, Viscoli CM, Horwitz RJ - Differences between women and men in survival after myocardial infarction: biology or methodology ? JAMA 1990; 263: 1092-6.

3. Dittrich H, Gilpin E, Nicod P, Cali G, Henning H, Ross J - Acute myocardial infarction in women:influence of gender on mortality and prognostic variables. Am J Cardiol 1988; 62: 1-7.

4. Gruppo Italiano per lo Studio della Streptoshinasi nell' Infarto Miocardico (GISSI) - Efectiveness of intravenous trombolytic treatment in acute myocardial infarction. Lancet 1986; 1: 397-402.

5. ISIS-2 - Randomized trial of intravenous streptoquinase, oral aspirin, both or neither among 17187 cases suspected acute myocardial infarction. Lancet 1988; 2: 349-60.

6. Passos LC, Lopes AA, Lessa I, Santos-Jesus R, Sanches A - Evolução da mortalidade por infarto agudo do miocárdio em Salvador. In Anais do IX Congresso Baiano de Cardiologia. Salvador-Bahia. Soc Cardiol Est Bahia, 1997: A-26.

7. Maynard C, Litwin PE, Martin JS, Weaver WD- Gender differences in the treatment and outcomes of acute myocardial infaction-results from the myocardial infartion triage and intervention registry. Arch Itern Med 1992; 152: 972-6.

8. Hammar N, Larsen F, Sanberg Eet al - Time trends in survival from myocardial infarction in Stolkholm Country 1976-1984, Int J Epidemiol 1992; 21: 1090.

9. Goldberg RJ, Gorak EJ, Yarzebski J et al - A community wide perspective of sex differences and temporal trends in the incidence and survival rates after acute myocardial infarction and out-of-hospital deaths caused by coronary heart disease.Circulation 1993; 87: 1947-55.

10. Vaccarino V, Krumholz HM, Berkman LF, Horwitz RI-Sex differences in mortality after myocardial infarction. Circulation 1995; 91: 1861-71.

11. Passos LC, Lopes AA, Souza CAM et al - Preditores de mortalidade hospitalar na era trombolítica para o infarto agudo do miocárdio em Salvador (Ba). Arq Bras Cardiol 1997; 68: 249-55.

12. Hosmer DW Jr, Lemeshow S - Applied Logistic Regression. NewYork: John Wiley \& Sons, 1989.

13. Norusis MJ - SPSS for Windows: Base System User's Guide, Release 6.0. Chicago: SPPS Inc, 1993.
14. Norusis MJ - SPSS for Windons: Advanced Statistics, Release 6.0. Chicago: SPSS Inc, 1993

15. Wilkinson $\mathrm{P}$, Laji K, Ranjadayalan $\mathrm{K}$ et al - Acute myocardial infarction in women: Survival analysis in first six months. Br Med J 1994; 309: 566.

16. White HD, Barbash GI, Modan M et al - After correcting for worse baseline caracteristics, women treated with thrombolytic terapy for acute myocardial infarction have the same mortality and morbidity as men except for higher incidence of hemorragic stroke. Circulation 1993; 88: 2097-103.

17. Tofler GH, Stone PH, Muller JE et al - Clinical manifestations of coronary heart disease in women. In: Eaker ED Packard B, Wenger NK, Clarkson TB, Tyroler HA, eds - Coronary Heart Disease in Women. Bethesda, Md: National Heart, Lung, and Blood Institute, National Institute of Health; 1987: 215-21.

18. Marrugat J, Antó JM, Sala J, Masiá R and REGICOR investigators - Influence of gender in acute and long-term cardiac mortality after a first myocardial infarction. J Clin Epidemiol 1994; 47: 111-8.

19. Lincoff MA, Callif RM, Ellis SG, Sigmon KN, Lee K, Leimberger JD Topol Ee t TAMI Study Group - Thrombolytic therapy for women with myocardial infarction: Is There a gender gap? J Am Coll Cardiol 1993; 22: 1780-7.

20. Diver D, Bier JD, Ferreira PE et TIMI-IIIA Investigators - Clinical and arteriographic characterization of patients with unstable angina without critical coronary arterial narrowing (from the TIMI-IIIA trial). Am J Cardiol 1994; 74: 531-7.

21. Clarke W, Gray D, Keating NA, Hamptom JR - Do women with acute myocardial infartion receive the same treatment as men ? Br Med J 1994; 309: 563.

22. Maynard C, Althose R, Cerqueira M, Olsulfka M, Kennedy JW Underutilization of trombolytic therapy in elegible women with acute myocardial infarction. Am J Cardiol 1991; 68: 529-30.

23. Kostis JB, Wilson AC, O'Dowd Ket al - Sex differences in the management and long-term outcome of acute myocardial infarction. Circulation 1994; 90: 171530 .

24. HerholzH, GoffDC, Ramsey DJ etal-Women and mexican americans receive fewer cardiovascular drugs following myocardial infarction than men and nonhispanic whites: the corpus christi heart project, 1988-1990. J Clin Epidemiol 1996; 49: 279-87.

25. Passos LC, Lopes AA, Fabiano MOS, Esteves FP, Félix MGF, Janos LF - Efeito da angioplastia primária na letalidade hospitalar do infarto agudo do miocárdio em mulheres. Arq. Bras Cardiol 1997; 69(supl I): 124 